\title{
THE STRUCTURE OF A GROUP OF PERMUTATION POLYNOMIALS
}

\author{
GARY L. MULLEN and HARALD NIEDERREITER
}

(Received 24 November 1983; revised 31 January 1984)

Communicated by R. Lidl

\begin{abstract}
Let $G_{q}$ be the group of permutations of the finite field $F_{q}$ of odd order $q$ that can be represented by polynomials of the form $a x^{(q+1) / 2}+b x$ with $a, b \in F_{q}$. It is shown that $G_{q}$ is isomorphic to the regular wreath product of two cyclic groups. The structure of $G_{q}$ can also be described in terms of cyclic, dicyclic, and dihedral groups. It also turns out that $G_{q}$ is isomorphic to the symmetry group of a regular complex polygon.
\end{abstract}

1980 Mathematics subject classification (Amer. Math. Soc.): 12 C 05, 20 B 25, 20 E 22, 51 F 15

\section{Introduction}

Let $F_{q}$ be the finite field of order $q$. Then every mapping from $F_{q}$ into itself can be uniquely represented by a polynomial in $F_{q}[x]$ of degree less than $q$, and composition of mappings corresponds to composition of polynomials $\bmod \left(x^{q}-x\right)$ (see [9, Chapter 7]). In particular, every group of permutations of $F_{q}$ can be represented by a set of polynomials in $F_{q}[x]$ of degree less than $q$ that is closed under composition mod $\left(x^{q}-x\right)$. According to a well-known definition (see [8, Chapter 4], [9, Chapter 7]), a polynomial $f$ over $F_{q}$ for which the corresponding polynomial mapping $c \in F_{q} \rightarrow f(c)$ is a permutation is called a permutation polynomial of $F_{q}$. Numerous papers have been written on the structure of permutation groups represented by a given group of permutation polynomials of $F_{q}$ under composition $\bmod \left(x^{q}-x\right)$; see for example Carlitz [1], 
Fryer [6], Lausch and Nöbauer [8, Chapter 4], Lidl and Niederreiter [9, Chapter 7], Nöbauer [12], and Wells [15], [16].

In the present paper we determine the structure of a group of permutation polynomials that was discovered recently by Niederreiter and Robinson [11]. In Remark 2 on page 205 of that paper it is pointed out that for odd $q$ the set of polynomials in $F_{q}[x]$ of the form $a x^{(q+1) / 2}+b x$ with $a, b \in F_{q}$ is closed under composition $\bmod \left(x^{q}-x\right)$. In particular, the set of permutation polynomials of $F_{q}$ of this form is a group under composition $\bmod \left(x^{q}-x\right)$, and we shall denote this group by $G_{q}$. We will establish some preparatory results in Section 2. These will enable us to determine the structure of $G_{q}$ in Section 3. In fact, several descriptions of the structure of $G_{q}$ will be given. We are grateful to the referee for pointing out that $G_{q}$ can also be described in terms of wreath products.

It is convenient to identify a polynomial over $F_{q}$ with the corresponding polynomial mapping, so that an identity $f=g$ with $f, g \in F_{q}[x]$ means $f \equiv g$ $\bmod \left(x^{q}-x\right)$. Throughout the rest of this paper, $q$ will be an odd prime power and $n$ will denote the value $(q-1) / 2$. The group $G_{q}$ can then be described as the group of permutations of $F_{q}$ of the form $a x^{n+1}+b x$ with $a, b \in F_{q}$.

\section{Preparatory results}

We determine first the order of the group $G_{q}$. We write $|G|$ for the order of a finite group $G$.

LEMMA $1 .\left|G_{q}\right|=2 n^{2}$.

Proof. Let $N$ be the number of permutations of $F_{q}$ of the form $f(x)=x^{n+1}+$ $b x$ with $b \in F_{q}$. Clearly, $f(x)$ is a permutation of $F_{q}$ if and only if $a f(x)$ is a permutation for $a \in F_{q}, a \neq 0$. If $a \neq 0$ is fixed, then the set of polynomial mappings $a x^{n+1}+b x$ with $b \in F_{q}$ also contains exactly $N$ permutations. If $a=0$, then $b x$ is a permutation if and only if $b \neq 0$. It follows that

$$
\left|G_{q}\right|=(q-1) N+q-1=(q-1)(N+1)=2 n(N+1) .
$$

By Theorem 5 of [11], $x^{n+1}+b x$ is a permutation polynomial of $F_{q}$ if and only if $\psi\left(b^{2}-1\right)=1$, where $\psi$ is the quadratic character defined by $\psi(0)=0$ and $\psi(c)=1$ or -1 depending on whether $c$ is a nonzero square or a nonsquare in $F_{q}$. 
Consequently,

$$
\begin{aligned}
N & =\sum_{\substack{b \in F_{q} \\
b \neq \pm 1}} \frac{1}{2}\left[1+\psi\left(b^{2}-1\right)\right]=-1+\frac{1}{2} \sum_{b \in F_{q}}\left[1+\psi\left(b^{2}-1\right)\right] \\
& =\frac{q-2}{2}+\frac{1}{2} \sum_{b \in F_{q}} \psi\left(b^{2}-1\right)=\frac{q-3}{2}=n-1,
\end{aligned}
$$

where we used Theorem 5.48 in [9] to evaluate the character sum. The lemma follows now from (1).

In order to determine the structure of $G_{q}$, we make use of the following law of composition observed in [11, p. 205]: if $f_{1}(x)=a x^{n+1}+b x$ and $f_{2}(x)=c x^{n+1}+$ $d x$ with $a, b, c, d \in F_{q}$, then

$$
\left(f_{1} \circ f_{2}\right)(x)=(a e+b c) x^{n+1}+(a h+b d) x,
$$

where $\circ$ denotes composition and

$$
e=\frac{1}{2}(c+d)^{n+1}+\frac{1}{2}(d-c)^{n+1}, \quad h=\frac{1}{2}(c+d)^{n+1}-\frac{1}{2}(d-c)^{n+1} .
$$

We construct now a special element of $G_{q}$ which will preve useful in the sequel. We recall that a generator of the cyclic multiplicative group of $F_{q}$ is called a primitive element of $F_{q}$.

LEMMA 2. Let $r$ be a primitive element of $F_{q}$. Then

$$
f(x)=\frac{1}{2}\left(1-r^{2}\right) x^{n+1}+\frac{1}{2}\left(1+r^{2}\right) x
$$

is an element of $G_{q}$ of order $n$.

Proof. For $g(x)=a x^{n+1}+b x$ with $a, b \in F_{q}$ we calculate $g \circ f$. The appropriate values of $e$ and $h$ from (3) are $e=\frac{1}{2}\left(1+r^{2}\right)$ and $h=\frac{1}{2}\left(1-r^{2}\right)$, so that (2) yields

$$
(g \circ f)(x)=\frac{1}{2}\left[a\left(1+r^{2}\right)+b\left(1-r^{2}\right)\right] x^{n+1}+\frac{1}{2}\left[a\left(1-r^{2}\right)+b\left(1+r^{2}\right)\right] x .
$$

A straightforward induction on $m$ shows then that the $m$-fold composition $f^{m}$ is given by

$$
f^{m}(x)=\frac{1}{2}\left(1-r^{2 m}\right) x^{n+1}+\frac{1}{2}\left(1+r^{2 m}\right) x .
$$

It follows that $f^{m}$ is the identity mapping if and only if $r^{2 m}=1$, and since the order of $r$ is $2 n$, the least positive $m$ for which $f^{m}$ is the identity mapping is $m=n$. In particular, $f$ is a permutation of $F_{q}$ and thus an element of $G_{q}$.

Let $r$ be a fixed primitive element of $F_{q}$ and let $X$ denote the element of $G_{q}$ constructed in Lemma 2. Furthermore, let $Y$ be the element of $G_{q}$ given by the linear permutation polynomial $r x$ of $F_{q}$. This notation will be used throughout the rest of this section. 
LEMMA 3. Every element of $G_{q}$ can be represented uniquely in the form $X^{i} Y^{j}$ with $0 \leqslant i<n, 0 \leqslant j<2 n$.

Proof. Since $\left|G_{q}\right|=2 n^{2}$ by Lemma 1 , it suffices to show that the elements $X^{i} Y^{j}, 0 \leqslant i<n, 0 \leqslant j<2 n$, are all distinct. Suppose $X^{i} Y^{j}=X^{k} Y^{l}$ with $0 \leqslant i$, $k<n$ and $0 \leqslant j, l<2 n$, where we can assume (with no loss of generality) that $i \geqslant k$. With $m=i-k$ we get then $X^{m}=Y^{l-j}$, so that $X^{m}$ is represented by a linear polynomial. The formula for $X^{m}$ in (4) shows that this is only possible if $r^{2 m}=1$. Since $0 \leqslant m<n$, it follows that $m=0$, hence $i=k$. Then $Y^{j}=Y^{\prime}$, and since $Y$ is an element of order $2 n$, we get $j=l$, and the proof if complete.

The following lemma gives a set of generators and relations for the group $G_{q}$. The symbol 1 will henceforth also denote the identity element of a group. The correct interpretation of the symbol 1 will always be clear from the context.

LEMMA 4. $G_{q}=\left\langle X, Y \mid X^{n}=Y^{2 n}=\left(X^{-1} Y\right)^{2}=1, X Y^{2}=Y^{2} X\right\rangle$.

Proof. The fact that $X$ and $Y$ generate $G_{q}$ follows already from Lemma 3. Now $X^{n}=1$ follows from Lemma 2 and $Y^{2 n}=1$ is clearly satisfied. Moreover, $X^{-1}=X^{n-1}$ is represented by

$$
\frac{1}{2}\left(1-r^{2(n-1)}\right) x^{n+1}+\frac{1}{2}\left(1+r^{2(n-1)}\right) x=\frac{1}{2}\left(1-r^{-2}\right) x^{n+1}+\frac{1}{2}\left(1+r^{-2}\right) x
$$

according to (4). Hence $X^{-1} Y$ and $Y^{-1} X$ are both represented by

$$
\frac{1}{2}\left(r^{-1}-r\right) x^{n+1}+\frac{1}{2}\left(r^{-1}+r\right) x
$$

since $r^{n}=-1$. This implies $\left(X^{-1} Y\right)^{2}=1$. The remaining relation $X Y^{2}=Y^{2} X$ can be checked easily.

On the basis of the relations in Lemma 4 we can calculate the group law for $G_{q}$.

\section{LEMMA 5.}

$$
\left(X^{i} Y^{j}\right)\left(X^{k} Y^{l}\right)= \begin{cases}X^{i+k} Y^{j+l} & \text { if } j \text { is even } \\ X^{i-k} Y^{j+l+2 k} & \text { if } j \text { is odd }\end{cases}
$$

Proof. The first part of (5) is clear since $Y^{2}$ commutes with $X$ by Lemma 4 . Next we note that $\left(Y X^{-1} Y^{-1}\right)^{-k}=Y X^{k} Y^{-1}$, and since $Y X^{-1} Y^{-1}=\left(Y X^{-1} Y\right) Y^{-2}$ $=X Y^{-2}$ by the third relation in Lemma 4 , we have

$$
Y X^{k}=\left(Y X^{-1} Y^{-1}\right)^{-k} Y=\left(X Y^{-2}\right)^{-k} Y=X^{-k} Y^{2 k+1} \text {. }
$$

The second part of (5) follows now, since for odd $j$ we get

$$
\begin{aligned}
\left(X^{i} Y^{j}\right)\left(X^{k} Y^{l}\right) & =X^{i} Y^{j-1} Y X^{k} Y^{l}=\left(X^{i} Y^{j-1}\right)\left(X^{-k} Y^{2 k+1+1}\right) \\
& =X^{i-k} Y^{j+l+2 k}
\end{aligned}
$$

where we used the first part of $(5)$ in the last step. 


\section{The structure of the group}

We convert now the presentation in Lemma 4 into a simpler one. It is clear that $G_{q}$ is also generated by $X$ and $R=X^{-1} Y$. From Lemma 4 we have $R^{2}=1$, and the relation $X Y^{2}=Y^{2} X$ can be rewritten as $X(X R)^{2}=(X R)^{2} X$, or $(X R)^{2}=$ $(R X)^{2}$. From the fact that $X$ commutes with $(X R)^{2}$, one obtains easily by induction that $(X R)^{2 k}=\left(X^{k} R\right)^{2}$ for all positive integers $k$. In particular, the relation $Y^{2 n}=1$ in Lemma 4 follows. Hence $G_{q}$ has the presentation

$$
G_{q}=\left\langle X, R \mid X^{n}=R^{2}=1,(X R)^{2}=(R X)^{2}\right\rangle .
$$

Thus $G_{q}$ is the group $n[4] 2$ in the notation of Coxeter and Moser [4]. More generally, for any positive integer $m$ the group $m[4] 2$ is defined by

$$
m[4] 2=\left\langle X, R \mid X^{m}=R^{2}=1,(X R)^{2}=(R X)^{2}\right\rangle .
$$

The relation $(X R)^{2}=(R X)^{2}$ can also be interpreted to say that $X$ commutes with $R X R=R^{-1} X R=X^{R}$. It follows now from Theorem 5 in Johnson [7, Chapter 15] that the presentation of $m[4] 2$ is the same as the presentation of the regular wreath product $C_{m}$ wr $C_{2}$, where $C_{m}$ denotes the cyclic group of order $m$. Thus we have shown the following result.

THEOREM. The group $G_{q}$ of all permutations of $F_{q}$ of the form $a x^{n+1}+b x$ with $a$, $b \in F_{q}$ is isomorphic to the regular wreath product $C_{n}$ wr $C_{2}$, where $n=(q-1) / 2$. More generally, the group $m[4] 2$ is isomorphic to the regular wreath product $C_{m}$ wr $C_{2}$ for all positive integers $m$.

The groups $m[4] 2$ have been studied in the literature in connection with the theory of symmetries of regular complex polytopes (see [3]). In particular, as indicated by Shephard [13], [14], the group $m[4] 2$ can be viewed as the symmetry group of the complex polygon with $m^{2}$ vertices $\left(\theta_{1}, \theta_{2}\right)$, where $\theta_{1}$ and $\theta_{2}$ run independently through the complex $m$ th roots of unity. Further details regarding the precise definitions of regular complex polytopes and their groups of symmetries can be found in [3]. Crowe [5] gives an alternative interpretation of $m[4] 2$ as a group of equivalence classes of quaternion transformations. The groups $m[4] 2$ belong also to the family of complex reflection groups; see the paper of Cohen [2] in which the notation $G(m, 1,2)$ is used for $m[4] 2$.

For odd $m$ the group $m[4] 2$ has the direct product form $D_{m} \times C_{m}$, where $D_{m}$ is the dihedral group of order $2 m$; see [4, p. 78]. This fact can also be deduced from the description of $m[4] 2$ as the regular wreath product $C_{m}$ wr $C_{2}$. Indeed, Theorem 
7.1 of Neumann [10] shows that $C_{m}$ wr $C_{2}$ has a nontrivial direct product decomposition. An inspection of the proof of this theorem yields a direct factor $Q$ isomorphic to $C_{m}=\langle\alpha\rangle$ and a direct factor $P$ consisting of all pairs $(b, f)$ with $b \in C_{2}=\langle\beta\rangle$ and $f: C_{2} \rightarrow C_{m}$ being a mapping satisfying $f(1) f(\beta)=1$. Now $P$ is generated by $S=\left(\beta, f_{0}\right)$ and $T=\left(1, f_{1}\right)$, where $f_{0}(1)=f_{0}(\beta)=1, f_{1}(1)=\alpha$, $f_{1}(\beta)=\alpha^{-1}$, and $S$ and $T$ satisfy the relations $S^{2}=T^{m}=(S T)^{2}=1$, so that $P$ is isomorphic to $D_{m}$. If $m[4] 2$ is given by the presentation in Lemma 4 (with $n$ replaced by $m$ ), then the direct factors $P$ and $Q$ can be identified explicitly. Using the group law in Lemma 5, one verifies that $P=\left\{X^{-j} Y^{j}: 0 \leqslant j<2 m\right\}$ is a normal subgroup of $m[4] 2$ with generators $S=X^{-1} Y$ and $T=X^{-2} Y^{2}$ and relations $S^{2}=T^{m}=(S T)^{2}=1$, so that $P$ is isomorphic to $D_{m}$. Furthermore, $Q=\left\langle Y^{2}\right\rangle$ is a normal cyclic subgroup of $m[4] 2$ of order $m$, and $P \cap Q=\{1\}$ since $m$ is odd. Moreover, $|P Q|=|P||Q|=2 m^{2}$, the order of $m[4] 2$, hence $m[4] 2$ is isomorphic to $P \times Q$. In particular, $G_{q}$ is isomorphic to $D_{n} \times C_{n}$ with $n=$ $(q-1) / 2$ provided that $q \equiv 3(\bmod 4)$.

For even $m$ the group $m[4] 2$ can also be described in terms of cyclic and dicyclic groups. Let $C_{2 m}=\langle\gamma\rangle$ be an abstract cyclic group of order $2 m$, and let

$$
E_{m}=\left\langle\delta, \varepsilon \mid \delta^{m}=\varepsilon^{2}=(\delta \varepsilon)^{2}\right\rangle
$$

be an abstract dicyclic group of order $4 m$ with generators $\delta$ and $\varepsilon$ of orders $2 m$ and 4, respectively (compare with [4]). Then $C_{2 m}$ has the subgroup $C_{m}=\left\langle\gamma^{2}\right\rangle$ of index 2 , and $E_{m}$ contains the dicyclic group

$$
E_{m / 2}=\left\langle\delta^{2}, \varepsilon \mid\left(\delta^{2}\right)^{m / 2}=\varepsilon^{2}=\left(\delta^{2} \varepsilon\right)^{2}\right\rangle
$$

as a subgroup of index 2. Hence $C_{m} \times E_{m / 2}$ is a normal subgroup of $C_{2 m} \times E_{m}$, and $H_{m}=L_{m}\left(C_{m} \times E_{m / 2}\right)$ is a subgroup of $C_{2 m} \times E_{m}$, where $L_{m}$ is the cyclic subgroup of $C_{2 m} \times E_{m}$ generated by $\left(\gamma, \delta^{-1}\right)$. The elements of $H_{m}$ can be represented uniquely in the form $\left(\gamma^{2 a+d}, \delta^{2 b-d_{\varepsilon}} \varepsilon^{c}\right)$ with $0 \leqslant a<m, 0 \leqslant b<m$, $0 \leqslant c<2,0 \leqslant d<2$. One constructs a mapping $\varphi: H_{m} \rightarrow m[4] 2$ by using the presentation of $m[4] 2$ in Lemma 4 (with $n$ replaced by $m$ ) and setting

$$
\varphi\left(\gamma^{2 a+d}, \delta^{2 b-d} \varepsilon^{c}\right)=X^{-2 b+d+m c / 2-c} Y^{2 a+2 b+c} .
$$

By an elementary but lengthy calculation based on the group law in Lemma 5 one shows that $\varphi$ is an epimorphism with kernel $K_{m}=\left\langle\left(\gamma^{m}, \delta^{m}\right)\right\rangle$. Therefore, $m[4] 2$ is isomorphic to $H_{m} / K_{m}$. This description of $m$ [4]2 for even $m$ is more explicit than the one given in Crowe [5]. 


\section{References}

[1] L. Carlitz, 'Permutations in a finite field', Proc. Amer. Math. Soc. 4 (1953), 538.

[2] A. M. Cohen, 'Finite complex reflection groups', Ann. Sci. Ecole Norm. Sup. (4) 9 (1976), $379-436$.

[3] H. S. M. Coxeter, Regular complex polytopes (Cambridge Univ. Press, London, 1974).

[4] H. S. M. Coxeter and W. O. J. Moser, Generators and relations for discrete groups (3rd ed., Springer-Verlag, Berlin-Heidelberg-New York, 1972).

[5] D. W. Crowe, 'The groups of regular complex polygons', Canad. J. Math. 13 (1961), 149-156.

[6] K. D. Fryer, 'Note on permutations in a finite field', Proc. Amer. Math. Soc. 6 (1955), 1-2.

[7] D. L. Johnson, Presentation of groups (London Math. Soc. Lecture Note Series, Vol. 22, Cambridge Univ. Press, Cambridge, 1976).

[8] H. Lausch and W. Nöbauer, Algebra of polynomials (North-Holland, Amsterdam, 1973).

[9] R. Lidl and H. Niederreiter, Finite fields (Encyclopedia of Math. and Its Appl., Vol. 20, Addison-Wesley, Reading, Mass., 1983).

[10] P. M. Neumann, 'On the structure of standard wreath products of groups', Math. Z. 84 (1964), 343-373.

[11] H. Niederreiter and K. H. Robinson, 'Complete mappings of finite fields', J. Austral. Math. Soc. (Ser. A) 33 (1982), 197-212.

[12] W. Nöbauer, 'Über eine Klasse von Permutationspolynomen und die dadurch dargestellten Gruppen', J. Reine Angew. Math. 231 (1968), 215-219.

[13] G. C. Shephard, 'Regular complex polytopes', Proc. London Math. Soc. (3) 2 (1952), 82-97.

[14] G. C. Shephard, 'Unitary groups generated by reflections', Canad. J. Math. 5 (1953), 364-383.

[15] C. Wells, 'Groups of permutation polynomials', Monatsh. Math. 71 (1967), 248-262.

[16] C. Wells, 'Generators for groups of permutation polynomials over finite fields', Acta Sci. Math. Szeged 29 (1968), 167-176.

Department of Mathematics

The Pennsylvania State University

University Park, Pennsylvania 16802

U.S.A.
Mathematical Institute Austrian Academy of Sciences

Dr. Ignaz-Seipel-Platz 2

A-1010 Vienna

Austria 